\title{
Fenología floral de la guaba (Inga edulis) en un valle interandino del Ecuador
} (Ice cream bean (Inga edulis) flower phenology
in Ecuadorian inter-Andean valley)

\author{
Maritza Quijia-Quijia ${ }^{1}$, Stephanie Castillo-Torres ${ }^{1}$, Wilson Vasquez-Castillo', Mauricio Racines-Oliva²
}

\begin{abstract}
Resumen
La investigación se realizó en la provincia de Pichincha, cantón Quito, parroquia Nayón, cuya temperatura varía entre $18^{\circ} \mathrm{C}$ a $22^{\circ} \mathrm{C}$ y se encuentra a una altitud de $2325 \mathrm{msnm}$. La guaba es una especie utilizada para brindar sombra en algunos cultivos como el café y el cacao. En este estudio se seleccionaron 15 árboles de guaba, en los cuales se marcaron 30 yemas vegetativas/planta, para seguirlas en el tiempo y observar su desarrollo. Se realizaron observaciones semanales del paso de las fenofases vegetativa a reproductiva del cultivo, para establecer la duración de cada una y la actividad térmica necesaria para que estos cambios se puedan dar.

Durante el estudio los árboles de guaba seleccionados presentaron brotación, caída del follaje y fructificación constante. Estos cambios variaron dependiendo de las condiciones climáticas de Nayón. Con los resultados obtenidos finalmente se determinó que la etapa de floración duró 155 días para el cultivo de guaba y un total de 497 unidades térmicas necesarias para cosechar frutos.

La escala BBCH (Biologische Bundesanstalt, Bundessortenamt und Chemische Industrie) describe el desarrollo fenológico de los cultivos desde la germinación hasta la senescencia; comprende 8 etapas principales que son la germinación, desarrollo de las hojas, formación de brotes, aparición de inflorescencias, floración, desarrollo de los frutos, maduración de frutos y senescencia. Con base a esta escala se desarrolló una descripción fenológica visual de la guaba de la fase de floración y desarrollo del fruto, con las subfases más importante visualizadas (521, 525, $529,621,625,629,723,725,729)$. Esta información básica y de gran utilidad constituye un fundamento clave para comprender mejor el proceso de desarrollo de este cultivo y mejorar las prácticas de campo y producción.
\end{abstract}

\section{Palabras clave}

Inga edulis; fenofases; BBCH; floración; fructificación.

\begin{abstract}
The research was carried out in the Nayón-Quito, Ecuadorian Pichincha-Province, with temperatures ranging between $18-22^{\circ} \mathrm{C}$, located at an altitude of 2325 meters above sea level. Ice cream bean is a species used to provide shade in some crops such as coffee and cocoa. In this study, 15 ice cream bean trees were selected in which 30 vegetative buds/plant were marked to follow them in time and observe their development. Weekly observations were made of the transition of the vegetative to reproductive structures of the crop in order to establish the duration of each stage presented by the plant as well as the thermal cumulation necessary for these changes to occur.

During the study, the selected trees selected presented constant bud break, foliage fall and fruiting. These changes varied according to the climatic conditions of Nayón. It was finally determined that ice cream bean flowering stage lasted 155 days and a total of 497 thermal units were needed to fruit harvest.

The BBCH scale (Biologische Bundesanstalt, Bundessortenamt und Chemische Industrie) describes the phenological development of crops from germination to senescence, comprising 8 main stages that are germination, leaf development, bud formation, inflorescence formation, flowering, fruit development, fruit ripening and senescence. Based on this scale a visual-phenological description of the ice cream bean fruit setting was obtained with the most relevant subphases presented by the plants $(521,525,529,621,625,629,723,725,729)$. This information constituted basic and outmost useful and relevant data to better understand the process of development of this crop and improve field and production practices.
\end{abstract}

\section{Keywords}

Inga edulis; phenophases; BBCH; flowering; fructification.

1 Universidad de las Américas, Facultad de Ingenierías y Ciencias Aplicadas (FICA). Quito-Ecuador. (Maritza Quijía-Quijía, maritza.quijia@udla.edu.ec 0000-0002-6085-7255; Stephanie Castillo-Torres, myriam.castillo@udla.edu.ec 0000-00020611-6210; Wilson Castillo-Vasquez, wilson.vasquez@udla.edu.ec 0000-0002-2163-4243.

2 Universidad de las Américas, Facultad de Ingenierías y Ciencias Aplicadas (FICA). Quito-Ecuador. mauricio.racines@udla. edu.ec 0000-0003-4335-4311. Autor de correspondencia. 


\section{Introducción}

La guaba es un árbol de copa densa, aparasolada y ancha con ramificaciones, que alcanza hasta de $30 \mathrm{~m}$ de altura, con diámetros de 30 a $60 \mathrm{~cm}$ de tronco. Posee una corteza recta, cilíndrica y de color marrón claro. Sus hojas son compuestas paripinnadas, con una longitud de 15 a 25 $\mathrm{cm}$. Posee un raquis con glándulas en forma de cráter, en el cual se encuentran de 4 a 6 pares de foliolos opuestos. Presenta foliolos con base obtusa, aguda u oblonga de color verde oscuro. Las inflorescencias son racimos terminales que pueden medir entre 7 y $12 \mathrm{~cm}$ de diámetro polar. Las flores son hermafroditas, con ovario supero, de color blanco; llegan a medir entre 3.5 a 4 cm de diámetro polar. Posee un cáliz y corona tubular con 5 a 4 lóbulos, en los cuales existen numerosos estambres con filamentos filiformes que presentan un diámetro polar entre 3 a 4 cm (Falcao y Clement, 2000). Es originaria de Centroamérica, el Caribe y la Amazonía, así como de los bosques andinos. Esta planta se desarrolla en temperaturas que van desde los 15 a los $22^{\circ} \mathrm{C}$ (Silva, Rogez, Da Silva y Larondelle, 2013). La guaba o guabo se desarrolla mejor en suelos alcalinos y ácidos, sin embargo, el pH óptimo para el cultivo es superior a 4. Igualmente, esta planta puede soportar cierto grado de salinidad. Se adapta a precipitaciones entre $1000 \mathrm{~mm}$ a 1300 mm (Sobanski y Marques, 2014).

En América Latina la guaba es utilizada en la industria maderera y alimenticia. Dentro de su género se encuentran aproximadamente 300 especies de árboles neotropicales, entre los cuales la gran mayoría son utilizados en la agricultura. Alrededor de 50 de estas especies poseen frutos comestibles. De igual manera, 33 especies del género Inga son utilizadas en los cultivos perennes por la sombra que brindan (Silva, Rogez, Da Silva y Larondelle, 2013).

Inga edulis ayuda a controlar la fragmentación del suelo evitando la erosión, ya que toleran los suelos ácidos (Silva, Rogez, Da Silva y Larondelle, 2013). Anualmente al caer las hojas forman un matillo que ayuda a mantener la humedad en el suelo y suprime el crecimiento de arvenses (Sobanski y Marques, 2014).

El manejo del cultivo de guaba se realiza con densidades de siembra de 70 a 150 árboles por hectárea, debido a la cantidad de follaje; estos son sembrados en distancias de 12×12 m. Sin embargo, en algunos casos los productores después de 3 o 4 años deben remover algunos árboles para evitar competencia.

Para el desarrollo ideal de la guaba, las plántulas deben ser sembradas en un lugar que cumpla con las condiciones agroclimáticas de esta especie. Durante el primer año se debe eliminar las arvenses que crecen alrededor del cultivo con el fin de evitar competencias por luz, agua y nutrientes. Este procedimiento se debe realizar hasta que el follaje pueda producir sombra suficiente para de esta manera limitar el desarrollo de arvenses.

El árbol no se poda completamente, solo las ramas secas puesto que, si se cortan por completo las ramas, el árbol muere. Los raleos se realizan en épocas lluviosas para aprovechar la luz y de esta manera influir positivamente en la productividad de nudos en las ramas (Monro, Velarde, Flores et al., 2016).

Las plantas de guaba se han ido adaptando a las distintas condiciones de clima y suelo. Por lo tanto, la floración irá de la mano con las condiciones ambientales, a las que se encuentren expuestas, como luz, temperatura y nutrientes (García, 2008). El principal objetivo de la floración es la reproducción de una planta, por lo cual, cada planta se caracteriza por experimentar transformaciones o cambios muy notables en un tiempo determinado. Todos estos cambios tienen orígenes en procesos bioquímicos internos de la planta, que tienen relación con 
las condiciones ambientales. Estos procesos emiten señales que reciben los meristemos para que inicie la fase de reproducción. En el caso de los árboles se puede observar la brotación de yemas, las cuales pueden ser florales y foliares (García, 2008).

El desarrollo y crecimiento de la gran mayoría de las plantas dependen de la temperatura, ya que esta interviene en los cambios de cada estado fenológico. Durante el desarrollo y crecimiento suceden cambios en las plantas desde la germinación hasta la madurez, entre los cuales se encuentran la brotación, floración y desarrollo de frutos (Parra, Fischer y Chaves, 2014).

Las unidades térmicas, también llamadas grados-día-desarrollo, son índices utilizados para estimar el desarrollo de los cultivos, ya que los mismos pueden predecir el cambio de las diferentes fenofases, entre ellas, la madurez fisiológica. Las unidades térmicas son aplicadas a sistemas de producción, debido a que es una técnica unificada que expresa la acumulación de la temperatura en las diferentes etapas. Esto depende de factores bióticos y abióticos, resultando en parámetros específicos para su desarrollo (García et al., 2012). Por lo tanto, las unidades térmicas son un parámetro que permite estimar la duración de las etapas de las plantas, entre ellas la floración y cosecha. Estas adquieren gran relevancia en sistemas productivos intensivos, donde la planificación de las prácticas culturales es importante.

Este cultivo se ha olvidado por varios años debido a la falta de investigaciones. Es considerado como un cultivo tradicional en algunos países, sin embargo, la falta de conocimiento de los beneficios que posee tanto para la salud como para los demás cultivos han hecho que se lo deje olvidado. El tiempo avanza y cada vez surgen más investigaciones en las que se deben mencionar los beneficios y componentes químicos que posee este árbol frutal, así como el manejo adecuado del cultivo (Sinisterra, 2016).

La información de la fenología tanto visual como reproductiva de Inga edulis en los valles interandinos es muy limitada, por consecuencia el objetivo del presente trabajo fue evaluar los cambios fenológicos por los cuales atraviesa la planta en la etapa reproductiva, con el fin de comprender los mismos y facilitar una guía visual. La hipótesis planteada fue que las yemas vegetativas pueden convertirse en yemas reproductivas. La información recolectada durante los meses de estudio constituye la base para la implementación de prácticas agronómicas y de fitomejoramiento, las cuales se sustentan en la información térmica necesaria para los cambios fenológicos de las plantas.

La escala BBCH (Biologische Bundesanstalt, Bundessortenamt und Chemische Industrie), debido a su alto nivel de detalle, ha sido utilizada desde hace muchos años en todo el mundo para investigaciones prácticas en horticultura y agricultura. Esta escala describe el desarrollo fenológico de las plantas mono y dicotiledóneas, utilizando una codificación numérica de las fases de crecimiento (Meier, Bleiholder, Buhr et al., 2009). Esta descripción detallada es de gran importancia para que el agricultor la utilice como referencia y de esta manera mejore el manejo agronómico y así la productividad (Meier et al., 2009).

\section{Metodología}

\subsection{Ubicación del estudio, condiciones climáticas y material vegetal}

Las observaciones fenológicas fueron realizadas durante los meses de julio y noviembre de año 2018, en la provincia de Pichincha, cantón Quito, parroquia Nayón, que tiene una temperatura entre $18^{\circ} \mathrm{C}$ y $22^{\circ} \mathrm{C}$ y se encuentra ubicada a $2325 \mathrm{msnm}$. Se seleccionaron 15 árboles de 7 años, en cada uno se marcaron 30 yemas vegetativas, es decir, estructuras que presentaban las ca- 
racterísticas deseadas, principalmente el color (el cual debe ser verde oscuro), y el tamaño (que debe ser de aproximadamente 3 milímetros). Para documentar los cambios en las diferentes fases reproductivas, se registraron datos de diámetro polar y diámetro ecuatorial en cada una de las estructuras desde la yema latente, racimo floral, flores y desarrollo del fruto. Los cambios en el diámetro fueron medidos con la ayuda de un calibrador (TACTIX 245111) y registrados visualmente con una cámara fotográfica (Canon rebel T6i).

\subsection{Unidades térmicas}

Para calcular las unidades térmicas se obtuvieron datos de la temperatura del lugar de estudio que es un monocultivo. Para registrar estos cambios térmicos se utilizó un data logger (HOBO U23 Pro v2 Temp/RH ONSET). Con estas temperaturas se calcularon las unidades térmicas (UT) obtenidas con la ecuación 1, llamada método residual, en donde T máx es la temperatura máxima calculada en un día, T min es la temperatura mínima calculada en un día, y la T base, que es la temperatura mínima a la que el cultivo detiene su crecimiento. Una vez obtenidas las unidades térmicas se calculó las unidades térmicas acumuladas con la ecuación 2, donde, $\Sigma$ es el promedio de la suma de los eventos, n es el número de eventos calculados.

A partir de los datos recolectados de las mediciones obtenidas al final del estudio, se realizó una curva de crecimiento en un plano cartesiano como se indica en la figura 1.

Ecuación 1. Método residual (Villalpando y Ruiz, 1993)

$$
\text { Unidades Térmicas }=\Sigma \frac{\text { Tmáx-Tmin }}{2} \text {-Tbase }
$$

Ecuación 2. Cálculo de unidades térmicas acumuladas (Méndez, 2015)

$$
\text { Unidades Térmicas Acumuladas }=\Sigma G D 1 \rightarrow n
$$

\subsection{Características de la escala $\mathrm{BBCH}$}

La escala BBCH describe el ciclo completo de desarrollo de los cultivos dividiéndolos en etapas que van desde la 0 al 9, ordenadas ascendentemente. La etapa 0 representa la germinación, 1 desarrollo de hojas, 2 formación de brotes, 3 crecimiento del tallo longitudinal, 4 desarrollo vegetativo, 5 emergencia de las inflorescencias, 6 floración, 7 desarrollo del fruto, 8 maduración y 9 senescencia o muerte (Meier et al., 2009).

Para el desarrollo de la escala BBCH en el cultivo de guaba se utilizó la escala que describe a la familia Fabaceae, en la cual se tomó en cuenta 3 dígitos como se muestra en la tabla 1. El primer dígito desde la izquierda indica el estadio principal, en el medio el número de ciclos que posee el cultivo al año y en la derecha es la descripción de cada fase. Para este estudio se utilizó desde la fase estado 5 a la 7, que son la emergencia de la inflorescencia, es decir su brote principal hasta el inicio de la fructificación (Meier et al., 2009).

En la presente investigación se describió el desarrollo de la yema latente, botones florales, flores jóvenes que están en proceso de abrirse, y maduras que ya se encuentran abiertas y el desarrollo del fruto en el cultivo estudiado. 
Tabla 1. Codificación escala BBCH para guaba

\begin{tabular}{|l|l|}
\hline \multicolumn{1}{|c|}{ Estado } & \multicolumn{1}{c|}{ Descripción } \\
\hline 5 & Presencia de brotes florales, aún rodeado por hojas. \\
\hline 521 & Primeros botones afuera de las hojas. \\
\hline 525 & Primeros botones solos en el exterior con hojas cerradas. \\
\hline 529 & Varios botones en los racimos aún permanecen cerrados. \\
\hline 6 & Floración. \\
\hline 620 & Primeras flores descubiertas. \\
\hline 621 & Presencia de 1 a 2 flores abiertas en el primer racimo. \\
\hline 623 & Presencia de 3 flores abiertas en los racimos por planta. \\
\hline 625 & Floración completa: flores descubiertas, 5 racimos por cada planta. \\
\hline 627 & Descenso de floración. \\
\hline 629 & Termina la fase de floración. \\
\hline 7 & Desarrollo del fruto. \\
\hline 720 & Vainas iniciales alcanzan el largo inicial (“vaina plana”). \\
\hline 723 & $30 \%$ de las vainas alcanzan el largo final de la vaina. \\
\hline 725 & $50 \%$ de las vainas alcanzan el largo final de la vaina. \\
\hline 727 & $70 \%$ de las vainas alcanzan el largo final de la vaina. \\
\hline 729 & La gran mayoría de vainas alcanzan el largo final. \\
\hline
\end{tabular}

\subsection{Método estadístico}

Para la descripción e interpretación de datos de la floración e inicio de fructificación del cultivo de guaba se utilizó estadística descriptiva de cada una de las fases, para obtener la variación del desarrollo.

Con los datos numéricos recolectados del diámetro polar y ecuatorial, se realizó el cálculo de promedio, varianza, desviación estándar y coeficiente de variación.

\section{Resultados y discusión}

\section{Desarrollo de la yema latente}

A los 21 días de haber marcado las yemas en los árboles, se presentó la diferenciación de los meristemos, con la producción de estructuras vegetativas o reproductivas. Para esto los brotes tuvieron que acumular 46.39 unidades térmicas.

El diámetro polar de la yema latente fue de $3.29 \mathrm{~mm}$ y el ecuatorial de $3.62 \mathrm{~mm}$ al inicio del estudio. A medida que fue creciendo se transformó en yema hinchada, que presentó una estructura de mayor tamaño, cuyas medidas fueron $3.65 \mathrm{~mm}$ y $3.78 \mathrm{~mm}$ de diámetro polar y ecuatorial respectivamente, aproximadamente a los 15 días. Con estos resultados se puede indicar que la evocación floral inicia con cambios morfológicos en las estructuras. De yema latente a yema hinchada la planta necesitó acumular 46.39 unidades térmicas. Estas fases corresponden al inicio de la etapa 5 de la escala BBCH. Una vez que el crecimiento de la estructura floral sigue, la yema brotada fue identificada. Los datos estadísticos descriptivos se presentan en la tabla 2 
Tabla 2. Estadísticos del desarrollo y crecimiento de las yemas latentes y botones florales en el cultivo de guaba en el valle de Nayón

\begin{tabular}{|l|c|c|c|c|}
\hline \multirow{2}{*}{ Estadísticos } & \multicolumn{2}{|c|}{ Yema latente $(\mathrm{mm})$} & \multicolumn{2}{c|}{ Yema brotada (mm) } \\
\cline { 2 - 5 } & D. polar & D. ecuatorial & D. polar & D. ecuatorial \\
\hline Promedio $(\mathrm{mm})$ & 3.29 & 3.62 & 30 & 4.68 \\
\hline Valor mínimo $(\mathrm{mm})$ & 0.12 & 2.27 & 8.39 & 2.03 \\
\hline Valor máximo $(\mathrm{mm})$ & 5.10 & 5.01 & 46.12 & 6.77 \\
\hline Rango $(\mathrm{mm})$ & 4.96 & 2.74 & 37.73 & 4.74 \\
\hline Varianza & 1.54 & 1.53 & 1.34 & 1.12 \\
\hline Desviación estándar & 1.20 & 120. & 6.62 & 1.01 \\
\hline CV\% & 36.44 & 33.11 & 21.10 & 21.60 \\
\hline
\end{tabular}

Al analizar los datos estadísticos obtenidos, se ve que la variabilidad de los datos supera el $20 \%$ y, de acuerdo con Franco y Hidalgo (2003), la variabilidad se almacena en el genoma de las plantas y esto puede expresarse en ciertas características, que se pueden juntar en dos grupos: en el primero se expresa en las características fenotípicas, es decir, las que se pueden ver como el color, tamaño, morfología y arquitectura, entre otras. En el segundo grupo están las que no son visibles; entre ellas se encuentran los productos o procesos que se dan al interior de la planta y son identificados actualmente por técnicas de biología molecular realizadas en los bancos de germoplasma (Franco y Hidalgo, 2003).

En este estudio se observaron las características fenotípicas que dieron origen a la variabilidad; esto es, el tamaño, ya que algunas se desarrollaban más pronto que otras y tenían similar tamaño al inicio de la investigación tanto en diámetro polar como ecuatorial.

\section{Desarrollo de los botones florales de guaba}

Durante el estudio se observó un crecimiento lento de los racimos florales, desde yema hinchada hasta el alargamiento de peciolos para la formación de los botones florales en cada racimo. Este proceso se completó en 98 días, período en el cual acumuló 432,26 unidades térmicas, y alcanzó un diámetro polar que inicia en 10.25 mm hasta llegar a 46.98 mm. El diámetro ecuatorial máximo del racimo fue $6.47 \mathrm{~mm}$ (figura 1).

Figura 1. Incremento del diámetro polar y ecuatorial $(\mathrm{mm})$ de la inflorescencia de guaba en función de la acumulación de unidades térmicas en el Valle de Nayón ( $n=90)$

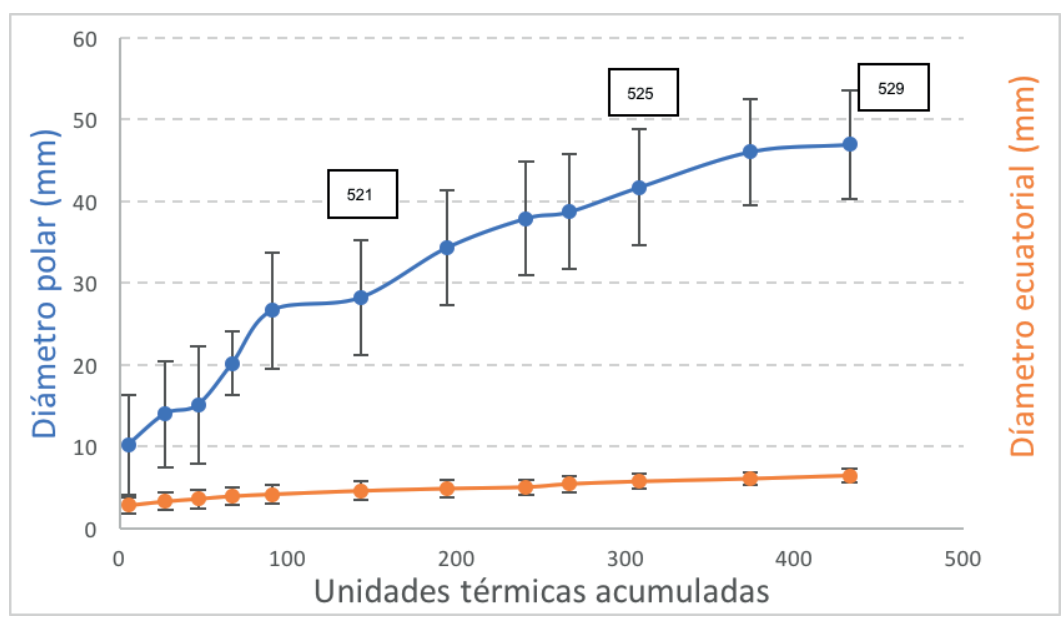


Estos resultados indican que conforme van creciendo longitudinalmente los botones florales, va aumentando el grosor del peciolo. Este cambio dependerá de la velocidad con la que se acumulen las unidades térmicas y, a su vez, con base en la temperatura diaria. Los desarrollos de los botones florales están considerados entre las etapas 521 y 529 de acuerdo con la escala $\mathrm{BBCH}$, como se puede evidenciar en la figura anterior.

También se pudo observar que los botones florales estaban rodeados por brácteas y su tamaño fue de diámetro polar $30 \mathrm{~mm}$ y ecuatorial $4.68 \mathrm{~mm}$ (etapa $529 \mathrm{BBCH}$ ), lo cual no se encuentra en el rango de medidas establecidas por Marín, Castaño y Gómez, (2012), en donde las medidas obtenidas fueron de $16 \mathrm{~mm}$ y $8 \mathrm{~mm}$ respectivamente. Esta variabilidad de datos se da ya que las características fenotípicas de las plantas, en cuanto al tamaño, fueron distintas.

Según Marín et al., (2012), cuando las brácteas que están cubriendo los botones florales se caen, se puede verificar que la inflorescencia tiene entre 6 y 28 botones florales. En el presente estudio se observó que los racimos tenían entre 20 y 25 botones florales, lo cual está dentro del rango indicado por Marín et al., (2012). La variación del número de racimos florales obtenidos se debió a la constante manipulación de las flores y las condiciones ambientales.

\section{Desarrollo de las flores del cultivo de guaba}

En este estudio se vio que una vez que el botón floral estuvo listo para abrirse, el tamaño se duplicó tanto en largo como en ancho, debido al despliegue rápido de la corola.

En la floración se observó que esta se divide en tres subfases como: 1) botón floral que es el estadio en el cual los órganos reproductivos (androceo y gineceo) se encuentran rodeados por los sépalos, 2) flor joven que hace referencia al estadio en el cual están visibles corola, cáliz, pistilos y estambres, y 3) flor madura (abierta) que se refiere al estadio en el que la flor se encuentra lista para la fecundación y el desarrollo del fruto; todo esto se obtuvo en la investigación realizada y es corroborado por lo descrito por Marín et al., (2012).

En este estudio se consideró las últimas 2 subfases de las tres descritas anteriormente, ya que se tomó en cuenta los datos de las flores jóvenes y maduras. Para llegar a esta etapa pasaron 41 días, en las que se acumuló 142.64 unidades térmicas para llegar a la antesis. En la figura 2, se muestra el crecimiento del diámetro polar y ecuatorial de las flores en y la acumulación de unidades térmicas.

Figura 2. Incremento del diámetro polar y ecuatorial $(\mathrm{mm})$ de los racimos florales de guaba desde botón hasta antesis, en función de la acumulación de unidades térmicas en el valle de Nayón (n=120)

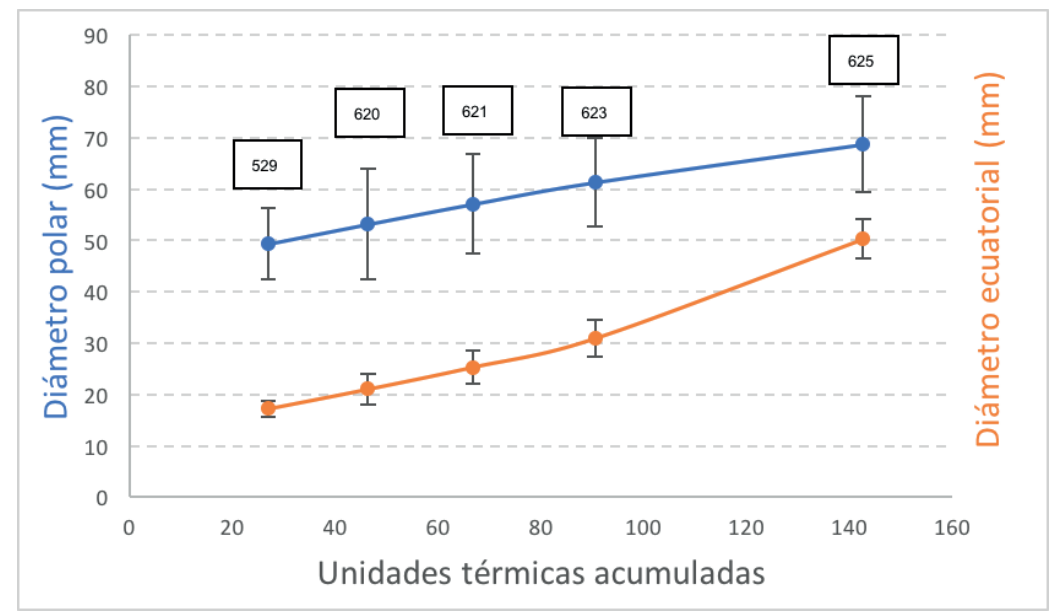


Las flores del cultivo de guaba se encontraban totalmente abiertas, previo a la fecundación y se observó que cambiaban de color y tamaño, puesto que la reducción en tamaño y la deshidratación de las estructuras florales se da una vez que se ha fecundado y comienza el desarrollo del fruto.

El crecimiento identificado de las flores se puede clasificar en las etapas de desarrollo 621,623 y 625 de acuerdo con la escala BBCH.

\section{Desarrollo del fruto}

Las flores cambiaron su color de blanco a café rojizo, por lo que se vio que ya habían sido fecundados e iniciaron un proceso de deshidratación seguido de la abscisión de estructuras. Estos cambios fueron concomitantes al desarrollo inicial de los frutos. Para que el fruto se desarrolle, se requirió de 43 días, durante los cuales acumuló 497.07 unidades térmicas. Las fases fenológicas evidenciadas en este período fueron clasificadas en la etapa 720, 723, 725, 727, 729 de la escala $\mathrm{BBCH}$. Todo el proceso de ontogenia de la fase reproductiva se encuentra descrito en tabla 3.

Tabla 3. Descripción de las fenofases del desarrollo de las estructuras reproductivas de la guaba en valle de Nayón

\begin{tabular}{|c|c|c|c|}
\hline $\begin{array}{l}\text { Código } \\
\text { BBCH }\end{array}$ & Descripción de fenofase & $\begin{array}{l}\text { Unidades térmicas } \\
\text { acumuladas (UTA) }\end{array}$ & $\begin{array}{l}\text { Letra correspon- } \\
\text { diente a figura } 3\end{array}$ \\
\hline 520 & $\begin{array}{l}\text { Inicio de etapa de floración. Yemas comienzan a } \\
\text { hincharse. }\end{array}$ & 0 & A \\
\hline 521 & Primeros capullos afuera de las hojas. & 5.29 & $B$ \\
\hline 525 & $\begin{array}{l}\text { Primeros capullos solos en el exterior de las hojas } \\
\text { cerradas. }\end{array}$ & 27.00 & C \\
\hline 529 & $\begin{array}{l}\text { Primeros pétalos visibles, en varios botones aún no } \\
\text { visibles. }\end{array}$ & 46.39 & $\mathrm{D}$ \\
\hline 620 & Primeras flores descubiertas. & 66.78 & $E$ \\
\hline 621 & Las flores abren en el primer racimo. & 90.49 & $\mathrm{~F}$ \\
\hline 623 & Las flores abren en 3 racimos por planta. & 142.64 & G \\
\hline 625 & $\begin{array}{l}\text { Floración completa: flores descubiertas, } 5 \text { racimos } \\
\text { por planta. }\end{array}$ & 193.84 & $\mathrm{H}$ \\
\hline 627 & Descenso de floración. & 240.66 & I \\
\hline 629 & Termina la fase de floración. & 266.17 & J \\
\hline 720 & $\begin{array}{l}\text { Vainas iniciales alcanzan el largo requerido ("vaina } \\
\text { completa"). }\end{array}$ & 307.73 & L \\
\hline 723 & 30 \% de las vainas alcanzan el largo final de la vaina. & 373.40 & L \\
\hline 725 & 50 \% de las vainas alcanzan el largo final de la vaina. & 432.26 & M \\
\hline 727 & $70 \%$ de las vainas alcanzan el largo final de la vaina. & 455.64 & $\mathrm{~N}$ \\
\hline 729 & $\begin{array}{l}>\text { al } 70 \% \text { de las vainas alcanzan el largo final de la } \\
\text { vaina. }\end{array}$ & 497.07 & 0 \\
\hline
\end{tabular}

Conjuntamente con la descripción de las fenofases, se encuentra en la tabla 3 una letra que corresponde a la imagen dentro de la figura 3, que se presenta a continuación. En esta figura se muestra la escala visual de la fenología reproductiva de la guaba, de acuerdo con la 
escala $\mathrm{BBCH}$, en la cual se puede evidenciar el cambio progresivo de las estructuras florales y el desarrollo del fruto.

Figura 3. Escala visual de las estructuras reproductivas de la planta de la guaba de acuerdo con la escala BBCH, en Nayón

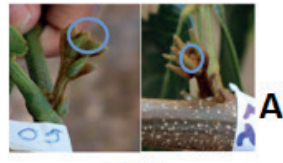

(520)

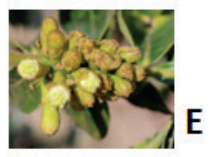

(620)

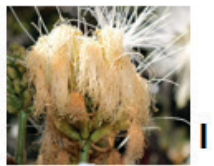

(627)

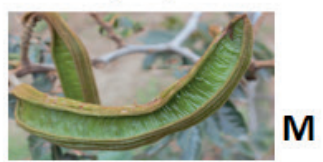

(725)

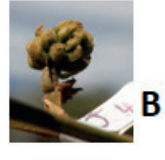

(521)

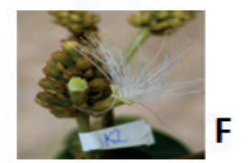

(621)

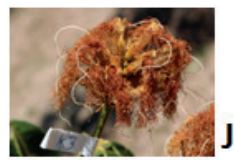

(629)

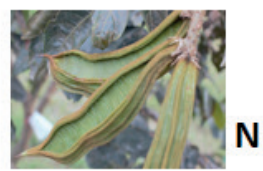

(727)

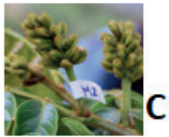

(525)

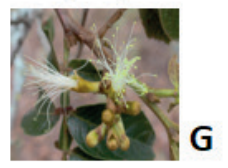

(623)

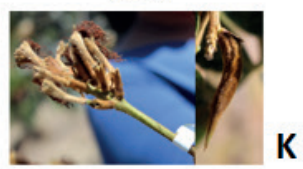

(720)

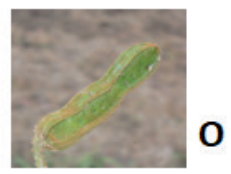

(729)

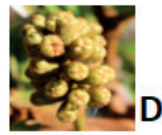

(529)

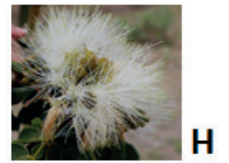

(625)

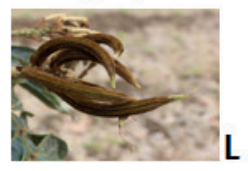

(723)

Se pudo observar que dentro de las flores que comprendían el racimo floral, en su interior contenían el fruto ya formado. Conforme este iba desarrollándose para llegar a su madurez fisiológica, el número de frutos por racimo iba disminuyendo y esto se debe, de acuerdo con Falcao y Clement, (2000), a que existe una competencia por los nutrientes necesarios para el crecimiento.

El proceso de floración y desarrollo del fruto es de gran importancia para la producción. En la floración hay cambios en estructuras; ciertos verticilos son descartados y otros cambian debido a la acción hormonal dentro del tejido (Marín, 2008). Durante ese período ocurren varios procesos como fecundación y desarrollo de fruto. De acuerdo con lo observado en el estudio y el conocimiento de las fenofases por las cuales atraviesa el cultivo, es posible hacer predicciones y labores culturales que favorezcan el desarrollo adecuado de los frutos, minimizando pérdidas.

\section{Conclusiones y recomendaciones}

En el presente estudio se llegó a determinar que existen 15 fenofases de desarrollo en el cultivo de guaba. El tiempo que tomó a las plantas fue de 127 días, desde el inicio de la aparición de los meristemas hasta la madurez fisiológica de los frutos; en este tiempo se acumularon 497,17 unidades térmicas.

Sobre la base de la información generada, se podrá hacer un uso más eficiente de las prácticas culturales, lo cual influirá de manera faborable a la productividad del cultivo de guaba.

Con el fin de ampliar la información presentada sobre este cultivo, se recomienda realizar un estudio fenológico de la etapa vegetativa, es decir, desde la germinación de la semilla hasta el trasplante-; debido a que existe escasa información en esta especie. 


\section{Agradecimientos}

El presente artículo forma parte del II Simposio Internacional de Producción Integrada de Frutas 2019, por lo cual los autores desean extender sus agradecimientos a los organizadores de este evento, que hace posible la extensión de conocimiento en frutales.

\section{Bibliografía}

Falcao, M. y Clement, C. (2000). Phenology and productivity of inga (Inga edulis) in Central Amazonia, Acta Amazonica, 8.

Franco, T. L. e Hidalgo, R. (2003). Análisis estadístico de datos de caracterización morfológica de recursos fitogenéticos. En Boletín Técnico, 8, Instituto Internacional de Recursos Fitogenéticos IPGRI (ISsue 8). Recuperado de https://www.bioversityinternational.org/fileadmin/_migrated/uploads/ tx_news/Anílisis_estadístico_de_datos_de_caracterización_morfológica_de_recursos_fitogenítiCos_894.pdf

García, D.; Morales, J.; Ardila, H.etal. (2012). Acumulación de grados-día en un cultivo de pepino (Cucumis sativus L.), en un Modelo de Producción Aeropónico. Phenology, Base Temperature, Physiological Time, Climate., 65(1), 6389-6398. doi: 10.1074/jbc.272.31.19304

García, J. (2008). La observación fenológica primaveral de los frutales, AEMET. 39-42.

Marín, O. H. (2008). Consumo de néctar por Aotus lemurinus y su rol como posible polinizador de las flores de Inga edulis (Fabales: Mimosoideae). Neotropical Pri-mates, 15(1), 30-32. doi: 10.1896/044.015.0108

Marín, O.; Castaño, A y Gómez, G. (2012). Fenología del guamo Inga edulis (Fabales: mimosoideae) en dos agroecosistemas del Quindía, Colombia. Recuperado el 1 de diciembre del 2018, de https:// www.researchgate.net/publication/242013826.

Meier, U.; Bleiholder, H.; Buhr, L. et al. (2009). The BBCH System to Coding the Phenological Growth Stages of Plants-history and Publications. Journal Für Kulturpflanzen, 61(2), 41-52. Recuperado el 23 de noviembre del 2018, de http://www.cabdirect.org/abstracts/20093092784.html

Méndez, C. (2015). Edad fisiológica de los cultivos: El uso de los grados días. Recuperado el 28 de septiembre del 2019, de http://www.mag.go.cr/bibliotecavirtual/av-1816.pdf.

Monro, A., R. Velarde, R. Flores, V. Soruco, J. Reyes, y W. Miliken. 2016. Manual agroforestería Inga. Royal Botanic Gardens, Kew \& Herencia, Chicago, IL, USA..

Parra, A.; Fischer, G. y Chaves, B. (2014). Thermal Time for Reproductive Phenological Stages of Pineapple Guava (Acca sellowiana (0. Berg) Burret). Acta Biológica Colombiana, 20(1), 163-173. doi: 10.15446/abc.v20n1.43390

Silva, E. M.; Rogez, H.; Da Silva, I. Q. y Larondelle, Y. (2013). Improving the De-sorption of Inga Edulis Flavonoids from Macroporous Resin: Towards a New Model to Concentrate Bioactive Compounds. Food and Bioproducts Processing, 91(4), 558-564. doi: 10.1016/j.fbp.2012.11.005

Sinisterra, R. M., Gallego, M. C., Armbrecht, I. (2016). Hormigas asociadas a nectarios extraflorales de árboles de dos especies de Inga en cafetales de Cauca. Colombia. Acta Agronómica. 65, 9-16.

Sobanski, N. y Marques, M. (2014). Effects of Soil Characteristics and Exotic Grass Cover on the Forest Restoration of the Atlantic Forest Region. Journal for Nature Conservation, 22(3), 217-222. doi: 10.1016/j.jnc.2014.01.001

Villalpando, J. y Ruiz, A. C. (1993). Observaciones agrometeorológicas y su uso en la agricultura. México: Editorial Lumusa. 